\title{
BMJ Open Regional health expenditure and health outcomes after out-of-hospital cardiac arrest in Japan: an observational study
}

\author{
Yusuke Tsugawa, ${ }^{1}$ Kohei Hasegawa, ${ }^{2}$ Atsushi Hiraide, ${ }^{3}$ Ashish K Jha ${ }^{1,4,5}$
}

To cite: Tsugawa $Y$, Hasegawa K, Hiraide A, et al. Regional health expenditure and health outcomes after out-of-hospital cardiac arrest in Japan: an observational study. BMJ Open 2015;5: e008374. doi:10.1136/ bmjopen-2015-008374

- Prepublication history and additional material is available. To view please visit the journal (http://dx.doi.org/ 10.1136/bmjopen-2015008374).

Received 31 March 2015 Revised 13 July 2015 Accepted 20 July 2015

CrossMark

For numbered affiliations see end of article.

Correspondence to Dr Yusuke Tsugawa; tsugawa@fas.harvard.edu

\section{ABSTRACT}

Objectives: Japan is considering policies to set the target health expenditure level for each region, a policy approach that has been considered in many other countries. The objective of this study was to examine the relationship between regional health expenditure and health outcomes after out-of-hospital cardiac arrest (OHCA), which incorporates the qualities of prehospital, in-hospital and posthospital care systems.

Design: We examined the association between prefecture-level per capita health expenditure and patients' health outcomes after OHCA.

Setting: We used a nationwide, population-based registry system of $\mathrm{OHCAs}$ that captured all cases with OHCA resuscitated by emergency responders in Japan from 2005 to 2011.

Participants: All patients with OHCA aged

1-100 years were analysed.

Outcome measures: The patients' 1-month survival rate, and favourable neurological outcome (defined as cerebral performance category 1-2) at 1-month.

Results: Among 618154 cases with $\mathrm{OHCA}$, the riskadjusted 1-month survival rate varied from 3.3\% (95\% Cl $2.9 \%$ to $3.7 \%)$ to $8.4 \%(95 \% \mathrm{Cl} 7.7 \%$ to $9.1 \%)$ across prefectures. The risk-adjusted probabilities of favourable neurological outcome ranged from $1.6 \%$ (95\% Cl $1.4 \%$ to $1.9 \%)$ to $3.7 \%$ (95\% Cl $3.4 \%$ to $3.9 \%$ ). Compared with prefectures with lowest tertile health expenditure, 1-month survival rate was significantly higher in medium-spending (adjusted OR $1.31,95 \% \mathrm{Cl} 1.03$ to $1.66, \mathrm{p}=0.03$ ) and highspending prefectures (adjusted OR 1.30, 95\% $\mathrm{Cl} 1.03$ to $1.64, p=0.02$ ), after adjusting for patient characteristics. There was no difference in the survival between medium-spending and high-spending regions. We observed similar patterns for favourable neurological outcome. Additional adjustment for regional per capita income did not affect our overall findings.

Conclusions: We observed a wide variation in the health outcomes after OHCA across regions. Lowspending regions had significantly worse health outcomes compared with medium-spending or highspending regions, but no difference was observed between medium-spending and high-spending regions. Our findings suggest that focusing on the median spending may be the optimum that allows for saving money without compromising patient outcomes.

\section{Strengths and limitations of this study}

- This is the first study that examined the association between regional health spending and the patient outcomes after out-of-hospital cardiac arrest (OHCA).

- We used a nationwide, population-based registry system of OHCAs that captured all cases with $\mathrm{OHCA}$ resuscitated by emergency responders in Japan.

- The outcomes after OHCA reflect a collective impact of prehospital, in-hospital and posthospital care systems, and thus they may be superior to the health outcomes used in previous studies that lean heavily on the quality of in-hospital care.

- Our study samples included only cases for which the emergency medical system was activated, resuscitation was attempted and the patients were transferred to the hospitals.

\section{INTRODUCTION}

Concerned about the rapid growth in health spending and the regional variation in health expenditure, the Japanese government is currently considering setting a target health expenditure level for each prefecture. ${ }^{1}$ While the specifics of this approach are not yet finalised, policymakers are considering using low-spending prefectures as potential benchmarks, or setting target health expenditure levels for each prefecture. These policies, which are analogous to ones proposed in other countries including the USA and other European countries, are controversial because many of these policies do not take into account quality of care or health outcomes in setting the target health expenditure level. ${ }^{2}$ If greater health expenditures are being used in helpful ways-in ways that improve quality and reduce poor outcomes, then policies that focus only on spending can potentially be harmful for the health of the population.

Regional variations in healthcare spending have been best studied in the USA ${ }^{34}$ and the 
evidence linking expenditures with outcomes has been mixed. ${ }^{5-8}$ Regional health spending can potentially impact a variety of health outcomes, including those at the community level and those within institutions such as hospitals. Outcomes after an out-of-hospital cardiac arrest (OHCA) are particularly salient because of three reasons: it is common (in the USA alone, an estimated 360000 people suffer from it annually), highly morbid (only $9.5 \%$ will survive to hospital discharge) ${ }^{9}$ and can serve as an indicator of health system performance more broadly. Outcomes after OHCA reflect a collective impact of prehospital, in-hospital and posthospital care systems, and inadequate performance of any part of this clinical chain could negatively impact the outcomes. Therefore, it can be a useful metric to assess the association between regional health expenditure and the population's health outcomes.

Given that many countries are struggling with rapidly rising health expenditure, understanding the relationship between health expenditure and health outcomes in Japan would provide important insights for other countries to examine their own strategies vis-à-vis spending and healthcare quality and outcomes. Therefore, in this study, we sought to answer three questions. First, how much variation is there in the outcomes after OHCA across 47 prefectures in Japan? Second, what is the relationship, if any, between per capita health expenditure at prefecture level and health outcomes after OHCA? Finally, given the strong policy concern that the most frugal regions may be achieving low spending by forgoing care for the oldest patients, is there any evidence that the relationship between health expenditure and health outcomes after OHCA varies by age group?

\section{METHODS}

\section{Study design and participants}

The All-Japan Utstein registry of the Fire and Disaster Management Agency (FDMA) is a nationwide, populationbased registry system of OHCAs in infants, children and adults, with Utstein-style data collection. ${ }^{10-12}$ All patients who had experienced non-traumatic OHCA and for whom resuscitation was attempted by emergency medical service (EMS) personnel with subsequent transport to hospitals from 1 January 2005 to 31 December 2011, with age of 1-100 years, were eligible for our analysis. We excluded those with age over 100 years from our analysis because the numbers were small and the differential proportion of people who do not request active life-saving procedures (ie, those people with "Do-not-resuscitate" (DNR) orders) across prefectures can potentially confound our inferences, and age is the strongest predictor of such decisions. ${ }^{13}$

Data were collected prospectively with an Utstein-style data form that included age, sex, aetiology of arrest, first documented cardiac rhythm, bystander's witness status, presence and type of cardiopulmonary resuscitation
(CPR) by bystander, and the use of a public-access automated external defibrillator (AED). Cardiac arrest was defined as the end of cardiac mechanical activity determined by the absence of signs of circulation. The aetiology of arrest was deemed cardiac unless evidence suggested trauma, respiratory diseases, cerebrovascular diseases, malignant tumours or any other non-cardiac cause. Attribution of cardiac or non-cardiac aetiology was made by the attending physicians in the emergency department in collaboration with the EMS personnel. Furthermore, the EMS personnel queried the medical control director at the hospital 1 month after the OHCA event to confirm the aetiology of the arrest. If there was a disagreement on the aetiology, the determination at 1-month was used. The study was approved by the Office of Human Research Administration at the Harvard School of Public Health. Informed consent was deemed unnecessary by the FDMA of Japan.

\section{Japanese healthcare system}

The population of Japan was roughly 128 million in 2010, with approximately 107 million people aged 18 years or older. ${ }^{14}$ Japan consists of 47 prefectures, which are the country's first jurisdiction and administrative division levels. The population size at each prefecture ranges from approximately 13 million in Tokyo to 600000 in Tottori. ${ }^{4}$ The land area and population size for each prefecture are listed in online supplementary appendix 1. The entire population is covered by the social health insurance system, and the prices and fees of the healthcare services are set uniformly regardless of the types and location of healthcare providers. The majority of healthcare providers are private, and the patients are free to choose which providers to visit. The coinsurance rate is fixed at $30 \%$ uniformly, except for the elderly and children. ${ }^{15}$ The municipal governments provide EMS through 802 fire stations with dispatch centres. The details about the EMS system in Japan have been described elsewhere. ${ }^{11}$

\section{Health outcomes}

The primary health outcome measure was 1-month survival after OHCA. The secondary outcome was favourable neurological outcome 1 month after cardiac arrest, which was defined as Glasgow-Pittsburgh cerebral performance category 1 (good performance) or 2 (moderate disability). ${ }^{10}$ The other categories-3 (severe cerebral disability), 4 (vegetative state) and 5 (death)were regarded as unfavourable neurological outcomes. This is the standard approach for the studies examining the neurological outcomes after OHCA. ${ }^{11}$

To collect follow-up data about survival and neurological status 1 month after the OHCA event, the EMS personnel who treated each patient with OHCA queried the medical control director at the hospital. Patient's neurological status was evaluated by the treating physician; the EMS received a written response. If the patient was not at the hospital, the EMS personnel conducted a 
follow-up search. Data forms were completed by EMS personnel in conjunction with the physicians who treated the patients, and the data were integrated into the Utstein registry system on the FDMA database server. Several regions developed additional local registry systems. In these areas, the information on each OHCA case was initially assembled using their data collection system. Then the information was exported and integrated into the FDMA database in which the data underwent further review. Forms were logic-checked by the computer system and were confirmed by the FDMA. If the data form was incomplete, the FDMA returned it to the respective fire station and the missing data were obtained.

\section{Per capita total health expenditure}

The information about annual total health expenditure per capita for each prefecture was extracted from the database created by the Ministry of Health, Labour and Welfare of Japan. ${ }^{16}$ The population data were available from the Statistics Bureau, Ministry of Internal Affairs and Communications. ${ }^{17}$ The total health expenditure was defined as the sum of inpatient and outpatient care, not including the expenditures due to dental care. Per capita total health expenditure was calculated by dividing total health expenditure by the population for each prefecture in 2005-2011. An exchange rate of 115 yen per US dollar was used for the analyses of health expenditure (as of 11 November 2014).

\section{Adjustment variables}

To account for differences in population characteristics across prefectures, we adjusted for demographic, clinical and response characteristics of the patients with OHCA. Demographic characteristics included age in 5-year increments (from $\geq 1$ year of age to 4,5 to 9 , and so on until 95 to 100), sex and the interaction between age and sex. Clinical characteristics consisted of aetiology of arrest (cardiac vs non-cardiac) and first documented rhythm (ventricular fibrillation (VF)/pulseless ventricular tachycardia vs other). The response characteristics included witnessed status (no witness, witnesses by layperson, witness by healthcare provider), type of bystander CPR (no bystander CPR, compression-only CPR, conventional CPR with chest compression and rescue breathing), and the use of a public-access AED by bystander (yes/no). We did not include the regional characteristics, such as EMS response time (which can be a proxy for a number of hospitals in a given region), because they are in the causal pathway linking the regional health spending and the health outcomes of patients.

\section{Statistical analysis}

We used these data to generate adjusted average values of each outcome in each prefecture. We pooled 7 years of data (2005-2011) and performed a person-level logistic regression for health outcomes. Each regression model included prefecture indicator variables, year indicators and the patient-level risk-adjustment variables listed above. The performance of the risk-adjustment model was evaluated using C-statistics (the prefecture indicators were excluded from the analysis when the C-statistics were calculated). ${ }^{18}$ The risk-adjusted outcomes were calculated using the predicted probabilities of outcomes for each patient with the distribution of covariates in our sample and the prefecture indicator set to that of each prefecture, and repeating the calculation across all 47 prefectures (also known as model-adjusted means, predictive margins or g-formula). ${ }^{19}$ SEs of the estimates were obtained by the delta method, and were used to calculate the $95 \%$ CIs. $^{19}$ Conceptually, this is equivalent to simulating the potential outcomes (counterfactuals) if all individuals with OHCA in our sample had been treated by the health system of a given prefecture.

We evaluated the association between the prefecture's per capita health expenditure and patients' health outcomes after OHCA. The prefectures were classified into three equal-sized groups (tertile) based on per capita health expenditure in order to address a potential nonlinear relationship between per capita health expenditure and health outcomes after OHCA (defined as lowspending, medium-spending and high-spending prefectures). In addition, we used per capita health expenditure as a continuous variable assuming a linear relationship between health expenditure and (log-odds of) health outcomes after OHCA. We used the personlevel data for our analysis in order to avoid ecological fallacy. ${ }^{21}$ In order to account for the potential clustering of cases with OHCA within each prefecture, we used generalised estimating equations (GEE) with binomial distribution, logit-link and an independent correlation structure. ${ }^{22-24}$ We used GEE instead of the mixed-effects models (also known as hierarchical models or multilevel models), because we were interested in the population average effects (estimated by GEE) rather than the subset-specific (individual-specific) effects (estimated by mixed-effects models). ${ }^{25}$ The regression models were adjusted for the year indicators, age, sex, the interaction between age and sex, aetiology of arrest (cardiac vs noncardiac), initial cardiac rhythm (VF or pulseless ventricular tachycardia (VT)), witness status, CPR by bystander, and use of public-access AED by bystander.

To evaluate the possibility that low-spending regions forgo spending on specific subpopulations, such as the oldest old population, we also examined the association between health spending and OHCA outcomes across three age groups: age $1-59,60-79$, and $80-100$. We fitted the same regression model as described above. We also fitted regression models with the interaction term between health expenditure and age group, and formally examined if the impact of health expenditure on outcomes after OHCA differs by age using the likelihood ratio test. We conducted a set of sensitivity analyses. First, we added the per capita income at prefecture level in 2011 (data extracted from the Japan Statistical 
Yearbook ${ }^{26}$ ) to the list of risk-adjustment variables in our regression models, as a measure of the socioeconomic status (SES) of the population. We did not include this variable in our primary analyses because the SES is a major determinant of access to healthcare and poor access to care is most likely a mediator for the relationship between spending and patient outcomes. Second, in order to evaluate if there is a plateau in the effect of regional health expenditure on health outcomes, we reanalysed the data using quintile of health expenditure instead of tertile. We also examined the association between regional health expenditure and the rate of return of spontaneous circulation (ROSC). Given that the rate of ROSC is a marker of the quality prehospital care, we aimed to investigate whether the difference in health outcomes across regions stems from the quality of prehospital care versus in-hospital and posthospital care systems. A two-sided $p$ value $<0.05$ was considered statistically significant. The GEE analysis was conducted using SAS, V.9.3 (SAS Institute, Cary, North Carolina, USA), and all other analyses were performed using Stata, V.12 (Stata-Corp, College Station, Texas, USA).

\section{RESULTS}

\section{Patient characteristics}

In the total catchment population of 128 million, 797422 OHCAs were reported from 1 January 2005 to
31 December 2011. From these, 145829 cases were excluded due to traumatic causes; 9657 were excluded as no resuscitation was attempted; and 6218 were excluded as the patients' age was less than 1 or higher than 100 years. Of the remaining cases with OHCA, 17547 cases with missing data on one of the covariates were excluded. Finally, 664 arrests were excluded from the analysis of the rate of favourable neurological outcome due to missing outcome data, leaving us with a final sample size of 618154 cases with OHCA for the analysis of 1-month survival rate and 617490 cases for the analysis of favourable neurological outcome (online supplementary appendix 2). Median age was 78 (IQR $67-85$ ), and $57.9 \%$ were men. Approximately two-thirds were due to cardiac causes, and VF or pulseless VT was observed as initial cardiac rhythm in $8.7 \%$ of the cases. Demographic, clinical and response characteristics of our sample, stratified by prefecture-level health expenditure, are presented in table 1.

\section{Regional variation in patients' health outcomes after OHCA}

Figure 1 and online supplementary appendix 3 show the variation in risk-adjusted outcomes of OHCA across prefectures. The C-statistics (area under the receiver operating characteristic (ROC) curve) were 0.81 for the risk-adjustment model for 1-month survival rate and 0.88

Table 1 Baseline characteristics of patients with out-of-hospital cardiac arrest, by prefecture-level per capita health expenditure

\begin{tabular}{|c|c|c|c|c|}
\hline & $\begin{array}{l}\text { Low-spending } \\
\text { prefectures }\end{array}$ & $\begin{array}{l}\text { Medium-spending } \\
\text { prefectures }\end{array}$ & $\begin{array}{l}\text { High-spending } \\
\text { prefectures }\end{array}$ & p Value \\
\hline Number of patients & $332213(53.7 \%)$ & $155077(25.1 \%)$ & $130864(21.2 \%)$ & \\
\hline \multicolumn{5}{|l|}{ Demographic characteristics } \\
\hline Age, median (IQR), year & $78(67-85)$ & $78(67-85)$ & $78(67-85)$ & $<0.01$ \\
\hline Male sex & $58.1 \%$ & $57.4 \%$ & $58.0 \%$ & $<0.01$ \\
\hline \multicolumn{5}{|l|}{ Clinical characteristics } \\
\hline Aetiology of arrest & & & & $<0.01$ \\
\hline Non-cardiac & $32.8 \%$ & $27.9 \%$ & $36.4 \%$ & \\
\hline Cardiac & $67.2 \%$ & $72.1 \%$ & $63.6 \%$ & \\
\hline $\begin{array}{l}\text { VF or pulseless VT as initial cardiac rhythm } \\
\text { Response characteristics }\end{array}$ & $8.3 \%$ & $8.9 \%$ & $9.3 \%$ & $<0.01$ \\
\hline Type of bystander-witness status & & & & $<0.01$ \\
\hline No witness & $58.2 \%$ & $56.7 \%$ & $58.5 \%$ & \\
\hline Layperson & $33.3 \%$ & $34.2 \%$ & $32.9 \%$ & \\
\hline Healthcare provider & $8.5 \%$ & $9.1 \%$ & $8.6 \%$ & \\
\hline \multicolumn{4}{|l|}{ CPR by bystander } & $<0.01$ \\
\hline No bystander CPR & $62.1 \%$ & $57.8 \%$ & $58.6 \%$ & \\
\hline Compression-only CPR & $26.7 \%$ & $28.2 \%$ & $28.4 \%$ & \\
\hline Conventional CPR & $11.2 \%$ & $13.9 \%$ & $13.0 \%$ & \\
\hline Use of public-access AED by bystander & $0.7 \%$ & $0.6 \%$ & $0.6 \%$ & $<0.01$ \\
\hline \multicolumn{5}{|l|}{ Prefecture-level characteristics } \\
\hline Per capita income (US\$) & $25343(3901)$ & $21827(2674)$ & $22764(1923)$ & $<0.01$ \\
\hline \multicolumn{5}{|c|}{$\begin{array}{l}\text { Samples are those cases with no missing data on all variables used in the regression analysis. Data are expressed as } n(\%) \text { for categorical } \\
\text { variables and mean (SD) for continuous variables, unless otherwise indicated. } p \text { Values are calculated using the } \chi^{2} \text { test for categorical } \\
\text { variables and ANOVA for continuous variables. Conventional CPR consists of chest compression and rescue breathing. } \\
\text { AED, automated external defibrillator; ANOVA, analysis of variance; CPR, cardiopulmonary resuscitation; VF, ventricular fibrillation; } \\
\text { VT ventricular tachycardia. }\end{array}$} \\
\hline
\end{tabular}


Figure 1 Risk-adjusted 1-month survival $(A)$ and favourable neurological outcome (B) after out-of-hospital cardiac arrest across prefectures. The $95 \% \mathrm{Cls}$ are shown in bars.
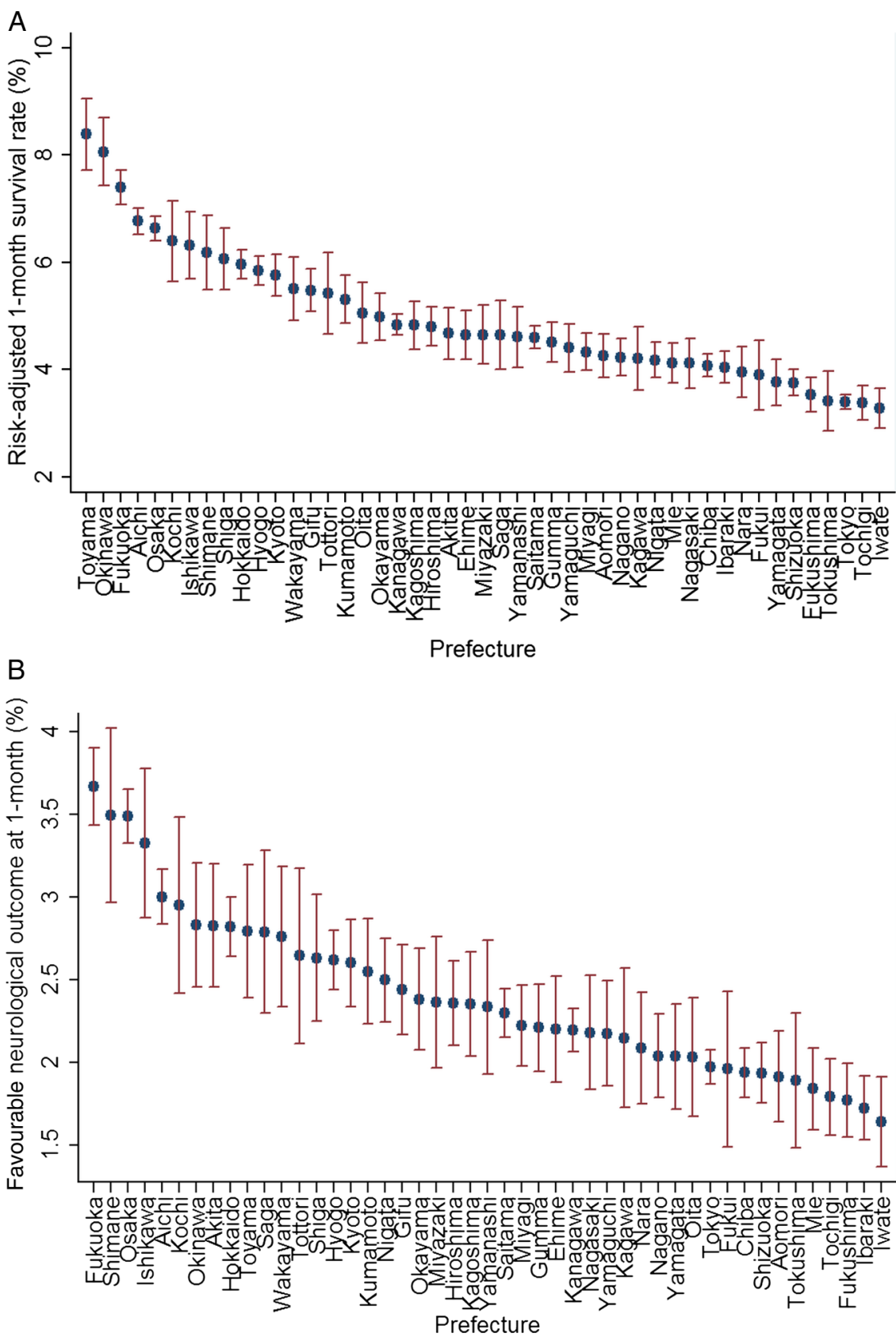

for the model for favourable neurological outcome, indicating the good discriminating power of our risk-adjustment models. ${ }^{18}$ The risk-adjusted 1-month survival rate ranged from $3.3 \%$ (95\% CI $2.9 \%$ to $3.7 \%$ ) in the Iwate prefecture to $8.4 \%(95 \%$ CI $7.7 \%$ to $9.1 \%)$ in the Toyama prefecture. Tokyo (the most populated prefecture in Japan) was the prefecture with one of the poorest risk-adjusted survival rate of $3.4 \%$ (95\% CI $3.3 \%$ to $3.5 \%$ ), whereas Osaka (the most populated prefecture in western Japan) exhibited one of the higher survival rates of $6.6 \%$ (95\% CI $6.4 \%$ to $6.9 \%)$.

The risk-adjusted probability of favourable neurological outcome varied from $1.6 \%(95 \%$ CI $1.4 \%$ to $1.9 \%$ ) in the Iwate prefecture to $3.7 \%$ (95\% CI $3.4 \%$ to $3.9 \%$ ) in the Fukuoka prefecture. Tokyo was again one of the poor-outcome prefectures with a $2.0 \%$ (95\% CI
$1.9 \%$ to $2.1 \%$ ) chance of experiencing good neurological outcome. In contrast, Osaka was one of the best with a $3.5 \%(95 \%$ CI $3.3 \%$ to $3.7 \%)$ chance of a favourable neurological outcome. Per capita health expenditure in 2005-2011 varied from US\$2504 (287 925 Japanese Yen) per year in the Kochi prefecture to US $\$ 1315$ (151 272 Japanese Yen) per year in the Saitama prefecture.

Association between prefecture-level health expenditure and patient health outcomes after OHCA

The relationships between the prefecture's per capita health expenditure and the risk-adjusted health outcomes after OHCA aggregated at prefecture level are shown in figure 2. The association between per capita health expenditure at prefecture-level and patient-level 

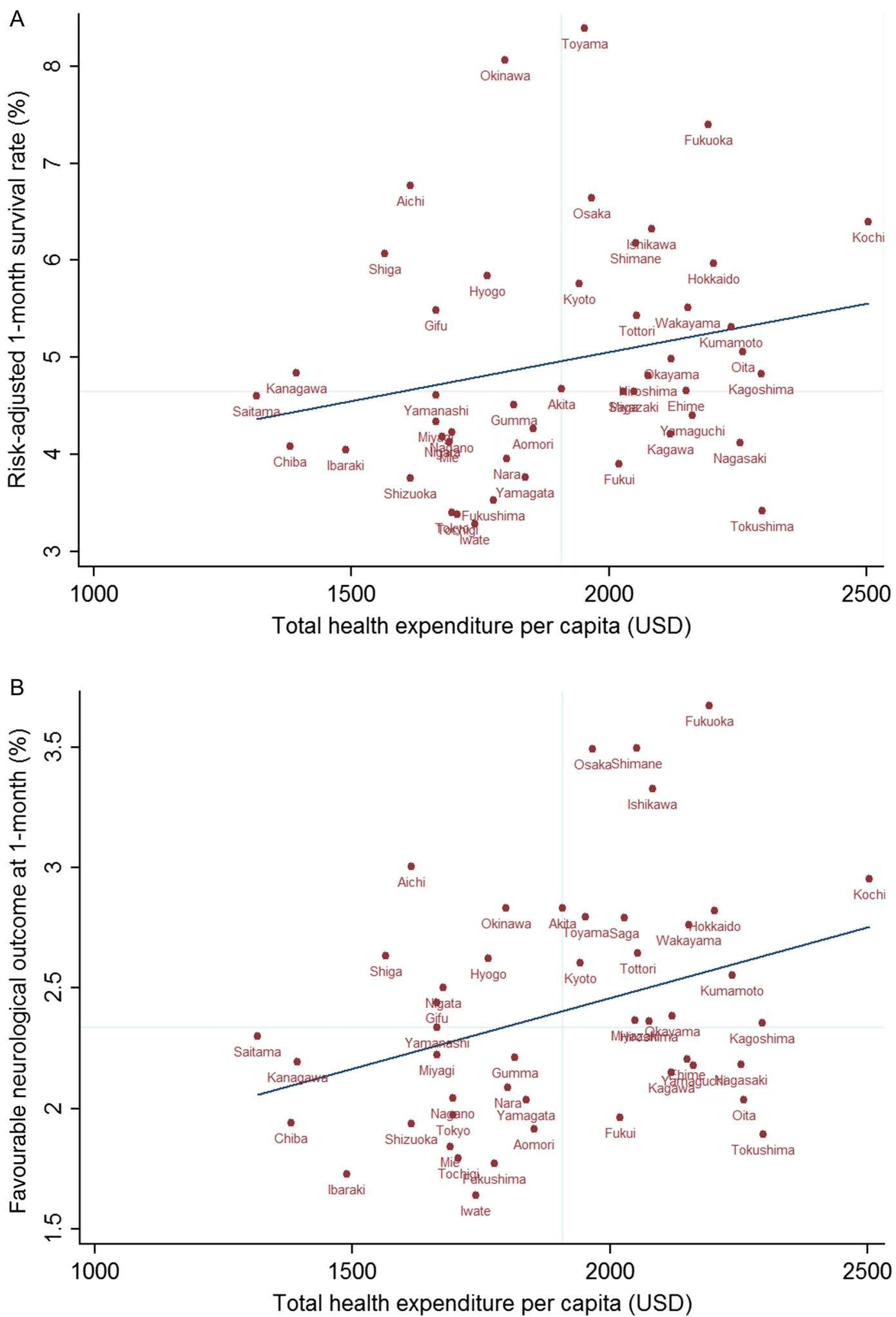

Figure 2 Association between total health expenditure per capita and risk-adjusted health survival $(A)$ and favourable neurological outcome (B) after out-of-hospital cardiac arrest at prefecture level.

outcomes after OHCA is presented in table 2. We found that a higher per capita health expenditure at the prefecture was associated with significantly better health outcomes after OHCA. For every US $\$ 100$ increase in per capita health expenditure at the prefecture, the patients with OHCA exhibited a 1.04 times higher odds of survival at 1 month (95\% CI 1.01 to $1.07, \mathrm{p}<0.01)$, and a 1.04 times higher odds of a favourable neurological outcome (95\% CI 1.02 to $1.07, \mathrm{p}<0.01$ ), after adjusting for patient risk (data not shown). 
Table 2 Association between per capita health expenditure at prefecture level and patients' health outcomes after out-of-hospital cardiac arrest

\begin{tabular}{|c|c|c|c|c|}
\hline \multirow[b]{2}{*}{ 1-Month survival rate } & \multicolumn{2}{|c|}{ Unadjusted (N=635 710) } & \multicolumn{2}{|l|}{ Adjusted $^{*}(\mathrm{~N}=618$ 154) } \\
\hline & OR (95\% Cl) & p Value & Adjusted OR (95\% Cl) & p Value \\
\hline \multicolumn{5}{|l|}{ Tertile of prefecture-level health expenditure per capita } \\
\hline Low & Ref & & Ref & \\
\hline Medium & $1.31(1.02$ to 1.67$)$ & 0.03 & $1.31(1.03$ to 1.66$)$ & 0.03 \\
\hline \multirow[t]{2}{*}{ High } & 1.30 (1.04 to 1.62$)$ & 0.02 & 1.30 (1.03 to 1.64$)$ & 0.02 \\
\hline & \multicolumn{2}{|c|}{ Unadjusted ( $\mathrm{N}=635$ 046) } & \multicolumn{2}{|l|}{ Adjusted $^{*}(\mathrm{~N}=617$ 490) } \\
\hline Favourable neurological outcome at 1 month & OR (95\% Cl) & p Value & Adjusted OR (95\% Cl) & p Value \\
\hline \multicolumn{5}{|c|}{ Tertile of prefecture-level health expenditure per capita } \\
\hline Low & Ref & & Ref & \\
\hline Medium & $1.30(1.02$ to 1.67$)$ & 0.04 & 1.29 (1.03 to 1.62$)$ & 0.03 \\
\hline High & $1.26(1.04$ to 1.53$)$ & 0.02 & $1.28(1.06$ to 1.55$)$ & 0.01 \\
\hline
\end{tabular}

The crude mean survival rate at 1 month after OHCA was $4.4 \%$ (95\% CI $4.3 \%$ to $4.4 \%)$ in low-spending prefectures, $5.7 \%$ (95\% CI $5.5 \%$ to $5.8 \%)$ in mediumspending prefectures, and $5.6 \%$ (95\% CI $5.5 \%$ to $5.7 \%$ ) in high-spending prefectures. The crude probabilities of favourable neurological outcome after OHCA was $2.1 \%$ (95\% CI $2.1 \%$ to $2.2 \%$ ) in low-spending prefectures, $2.8 \%$ (95\% CI $2.7 \%$ to $2.9 \%$ ) in medium-spending prefectures, and $2.7 \%$ (95\% CI $2.6 \%$ to $2.8 \%$ ) in highspending prefectures. Similar to the results of the linear regression analysis, compared with cases with OHCA in the prefectures with lowest tertile health expenditure, those in the medium-spending and high-spending prefectures exhibited significantly higher survival rates (table 2). The 1-month survival rate showed a 1.31 times higher odds (95\% CI 1.03 to $1.66, p=0.03$ ) in mediumspending prefectures, and a 1.30 times higher odds (95\% CI 1.03 to $1.64, \mathrm{p}=0.02$ ) in highest spending prefectures, compared with lowest spending prefectures. Likewise, the odds of a favourable neurological outcome were 1.29 times higher ( $95 \%$ CI 1.03 to $1.62, \mathrm{p}=0.03$ ) in medium-spending prefectures, and 1.28 times higher (95\% CI 1.06 to $1.55, \mathrm{p}=0.01$ ) in high-spending prefectures. We did not observe a significant difference in health outcomes between cases with OHCA in mediumspending and those in high-spending prefectures (data not shown). Additional adjustment for the prefecturelevel per capita income level did not qualitatively affect our overall findings (online supplementary appendix 4). Both medium-spending and high-spending regions had higher probabilities of favourable neurological outcomes and better survival compared with low-spending regions, although some of these differences were no longer statistically significant (even though the effect sizes were similar). The analysis using the quintile of regional health expenditure showed a positive association between regional spending and outcomes after OHCA; however, we did not observe a clear plateau effect in the relationship between regional health expenditure and health outcomes, probably due to the lack of statistical power to precisely make estimates (online supplementary appendix 5). We did not observe a significant relationship between regional health expenditure and the rate of ROSC, suggesting that low-spending regions had worse health outcomes mainly due to the lower quality of in-hospital and posthospital care systems rather than that of prehospital care (online supplementary appendix 6).

\section{Relationships between health expenditure and OHCA outcomes across different age groups}

We found that the relationships between health expenditure and OHCA outcomes were consistent across all three age groups (table 3). Compared with low-spending prefectures, both medium-spending and high-spending prefectures showed higher 1-month survival rates and higher probabilities of favourable neurological outcomes after OHCA. Although the statistical power is limited in a small number of metrics, we still observed higher odds of better OHCA outcomes in these prefectures. We observed a trend towards a stronger relationship among patients with OHCA aged 80-100, compared with younger age groups; however, the results of the likelihood ratio test did not show a statistically significant interaction between age group and health expenditure ( $p=0.30$ for survival and $\mathrm{p}=0.36$ for a favourable neurological outcome).

\section{DISCUSSION}

In the national study of patients with OHCA in Japan, we found more than a twofold variation in health outcomes after OHCA across prefectures. Our results showed that low-spending regions had significantly worse health outcomes after OHCA, compared with medium-spending or high-spending regions; however, 
Table 3 Adjusted association between per capita health expenditure at prefecture level and patients' health outcomes after out-of-hospital cardiac arrest, stratified by age group*

\begin{tabular}{|c|c|c|c|c|c|c|}
\hline \multirow[b]{2}{*}{ 1-Month survival rate } & \multicolumn{2}{|c|}{ Age $1-59(\mathrm{~N}=91$ 108) } & \multicolumn{2}{|c|}{ Age $60-79(\mathrm{~N}=250705)$} & \multicolumn{2}{|c|}{ Age $80-100(\mathrm{~N}=276$ 341) } \\
\hline & $\begin{array}{l}\text { Adjusted OR } \\
(95 \% \mathrm{Cl})\end{array}$ & p Value & $\begin{array}{l}\text { Adjusted OR } \\
(95 \% \mathrm{Cl})\end{array}$ & p Value & $\begin{array}{l}\text { Adjusted OR } \\
(95 \% \mathrm{Cl})\end{array}$ & p Value \\
\hline \multicolumn{7}{|c|}{ Tertile of prefecture-level health expenditure per capita } \\
\hline Low & Ref & & Ref & & Ref & \\
\hline Medium & 1.25 (1.01 to 1.55$)$ & 0.04 & $1.32(1.01$ to 1.71$)$ & 0.04 & $1.37(1.07$ to 1.74$)$ & 0.01 \\
\hline \multirow[t]{2}{*}{ High } & 1.29 (1.05 to 1.60$)$ & 0.02 & $1.26(0.99$ to 1.61$)$ & 0.06 & 1.39 (1.09 to 1.78$)$ & $<0.01$ \\
\hline & \multicolumn{2}{|c|}{ Age 1-59 (N=90 996) } & \multicolumn{2}{|c|}{ Age $60-79(\mathrm{~N}=250403)$} & \multicolumn{2}{|c|}{ Age $80-100(\mathrm{~N}=276$ 091) } \\
\hline $\begin{array}{l}\text { Favourable neurological } \\
\text { outcome at } 1 \text { month }\end{array}$ & $\begin{array}{l}\text { Adjusted OR } \\
(95 \% \mathrm{Cl})\end{array}$ & p Value & $\begin{array}{l}\text { Adjusted OR } \\
(95 \% \mathrm{Cl})\end{array}$ & p Value & $\begin{array}{l}\text { Adjusted OR } \\
(95 \% \mathrm{Cl})\end{array}$ & p Value \\
\hline \multicolumn{7}{|c|}{ Tertile of prefecture-level health expenditure per capita } \\
\hline Low & Ref & & Ref & & Ref & \\
\hline Medium & $1.24(0.98$ to 1.57$)$ & 0.07 & $1.30(1.02$ to 1.66$)$ & 0.04 & $1.37(1.10$ to 1.70$)$ & $<0.01$ \\
\hline High & $1.27(1.05$ to 1.54$)$ & 0.01 & $1.23(1.01$ to 1.50$)$ & 0.04 & $1.46(1.14$ to 1.86$)$ & $<0.01$ \\
\hline
\end{tabular}

the health outcomes of the high-spending regions were not better than those of the medium-spending regions. These relationships appeared to be stronger among the oldest age group (age 80-100) compared with younger age groups, although the formal interaction test was not statistically significant. These findings suggest that any policy interventions targeted towards healthcare costs alone and not taking into account health outcomes may have a detrimental effect on the population health, especially among the oldest.

While we found a positive association between regional health expenditure and health outcome after OHCA, the relationship was not linear. Low-spending prefectures exhibited worse health outcomes, but the health outcomes in high-spending prefectures were not better than those in medium-spending prefectures. This has two important policy implications. Setting the target spending level to lowest-spending group is not likely to be beneficial for the health of the population. However, more spending in higher spending areas might not lead to better outcomes either. Our findings indicate that a medium level of health expenditure can potentially rein in healthcare costs without compromising health outcomes of the population.

We are unaware of any prior study that has studied the relationship between health spending and outcomes after OHCA. Fisher et at studied the relationship between regional health spending and mortality rate among Medicare enrollees hospitalised for three common conditions in the USA. They found that higher regional spending was associated with a slightly higher risk of death for colorectal cancer and acute myocardial infarction (AMI), but had no impact on the mortality among patients with hip fracture. Baicker and Chandra ${ }^{7}$ conducted a state-level analysis and reported that states with higher Medicare spending had a lower quality of care, using process measures for treatment of six common conditions (AMI, breast cancer, diabetes, heart failure, pneumonia and stroke). These studies lean heavily on the quality of in-hospital care, in contrast to the outcomes after OHCA, which are affected by a quality of prehospital, in-hospital and posthospital care, collectively.

Even though there is no single health outcome metric that can comprehensively measure the performance of the regional health system, the OHCA outcomes have several advantages over other health outcomes. The health outcomes after OHCA reflect a broader performance of the regional health system including prehospital (immediate recognition of cardiac arrest and activation of the emergency response system, early CPR and rapid defibrillation), in-hospital (integrated postcardiac arrest care) and posthospital care systems (rehabilitation). As a consequence, the study of OHCA outcomes enabled us to evaluate a composite performance of different aspects of the healthcare delivery system. In addition, by focusing on both mortality and neurological outcome, we could evaluate the quality of services to keep patients alive, as well as the quality of care that help the clinical recover, which indeed is the ultimate goal of the health system for treating patients with OHCA. We found that regional health expenditure did not have a significant impact on the rate of ROSC, which indicates that lower regional spending had a detrimental effect on the outcomes after OHCA through a lower quality of in-hospital and posthospital care systems, rather than that of prehospital care.

Our study has several limitations. First, we could not assess why low-spending regions had worse outcomes. This is an important area for examination in future work. A second limitation is that our study samples included only cases for which the emergency medical 
system was activated, resuscitation was attempted, and the patients were transferred to the hospitals. Different prefectures may have different criteria for whether the patients with OHCA with low probabilities of survival are pronounced dead at the scene without transfer to hospitals. Similarly, the study population may include individuals who do not wish life-saving treatment (eg, individuals with DNR orders) such as those with advanced age, disabilities or late-stage cancer. It is also possible that the likelihood of making DNR orders is influenced by local norms and thus differs across prefectures. Third, the outcomes after OHCA may not capture the quality of outpatient care. Further research is warranted to evaluate if higher regional health spending leads to better quality of outpatient care. Finally, the integrity and validity of the data, and ascertainment bias, are potential source of bias. The use of uniform data collection based on Utstein-style guidelines for reporting and recording the cardiac arrest cases, the large sample size and the population-based design are expected to minimise these potential threats to validity.

In conclusion, we found more than twofold variations in OHCA outcomes across prefectures in Japan. We observed a non-linear relationship between regional health spending and patients' outcomes after OHCA. Low-spending regions had significantly worse health outcomes, but the health outcomes in high-spending regions were not better than those in medium-spending regions. Our findings indicate that setting health spending targets to the level of the lowest spending group may be harmful in terms of health outcomes, especially for emergency cases such as OHCA. The fact that more spending in higher spending areas does not appear to improve patient outcomes suggests that for national policymakers in countries wishing to set budget targets, focusing on the median spending may be the optimum that allows for saving money without compromising patient outcomes.

\section{Author affiliations \\ ${ }^{1}$ Department of Health Policy and Management, Harvard School of Public Health, Boston, Massachusetts, USA \\ ${ }^{2}$ Department of Emergency Medicine, Massachusetts General Hospital and Harvard Medical School, Boston, Massachusetts, USA \\ ${ }^{3}$ Department of Acute Medicine, Kinki University Faculty of Medicine, Osaka, Japan \\ ${ }^{4}$ Harvard Global Health Institute, 42 Church Street, Cambridge, MA 02138 ${ }^{5}$ Department of General Internal Medicine, Brigham and Women's Hospital and the Veterans Affairs Boston Healthcare System, Boston, Massachusetts, USA}

\section{Twitter Follow Ashish Jha at @ashishkjha}

Contributors YT is the guarantor for the integrity of the data and the accuracy of the data analysis. YT, AKJ and KH contributed in the study concept and design. $\mathrm{KH}$ and $\mathrm{AH}$ were involved in the acquisition of data. YT and AKJ participated in the analysis and interpretation of data. YT and AKJ drafted the manuscript. YT, AKJ, KH and AH performed the critical revision of the manuscript for important intellectual content. YT performed the statistical analysis.
Funding This research received no specific grant from any funding agency in the public, commercial or not-for-profit sectors.

Competing interests None declared.

Ethics approval Harvard School of Public Health, Office of Human Research Administration.

Provenance and peer review Not commissioned; externally peer reviewed.

Data sharing statement Please contact the authors for the statistical code and the data set.

Open Access This is an Open Access article distributed in accordance with the Creative Commons Attribution Non Commercial (CC BY-NC 4.0) license, which permits others to distribute, remix, adapt, build upon this work noncommercially, and license their derivative works on different terms, provided the original work is properly cited and the use is non-commercial. See: http:// creativecommons.org/licenses/by-nc/4.0/

\section{REFERENCES}

1. Nihon Keizai Shimbun. Curbing health expenditure, tackling high hospitalization costs, as large as 2.1 times difference in health expenditure between prefectures (in Japanese). 12 August 2014

2. Ministry of Health Labour and Welfare. Regional variation of health expenditure in Japan (in Japanese). Secondary regional variation of health expenditure in Japan (in Japanese) 2012. http://www.mhlw. go.jp/stf/seisakunitsuite/bunya/kenkou_iryou/iryouhoken/database/ iryomap/index.html

3. Chassin MR, Brook RH, Park RE, et al. Variations in the use of medical and surgical services by the Medicare population. $N$ Engl $J$ Med 1986;314:285-90.

4. Wennberg J, Gittelsohn A. Small area variations in health care delivery. Science 1973;182:1102-8.

5. Fisher ES, Wennberg DE, Stukel TA, et al. The implications of regional variations in Medicare spending. Part 1: the content, quality, and accessibility of care. Ann Intern Med 2003;138:273-87.

6. Fisher ES, Wennberg DE, Stukel TA, et al. The implications of regional variations in Medicare spending. Part 2: health outcomes and satisfaction with care. Ann Intern Med 2003;138:288-98.

7. Baicker K, Chandra A. Medicare spending, the physician workforce, and beneficiaries' quality of care. Health Aff (Millwood) 2004;(Suppl Web Exclusives):W4-184-97.

8. Landrum MB, Meara ER, Chandra A, et al. Is spending more always wasteful? The appropriateness of care and outcomes among colorectal cancer patients. Health Aff (Millwood) 2008;27:159-68.

9. Go AS, Mozaffarian D, Roger VL, et al. Heart disease and stroke statistics-2013 update: a report from the American Heart Association. Circulation 2013;127:e6-245.

10. Jacobs I, Nadkarni V, Bahr J, et al. Cardiac arrest and cardiopulmonary resuscitation outcome reports: update and simplification of the Utstein templates for resuscitation registries: a statement for healthcare professionals from a task force of the International Liaison Committee on Resuscitation (American Heart Association, European Resuscitation Council, Australian Resuscitation Council, New Zealand Resuscitation Council, Heart and Stroke Foundation of Canada, InterAmerican Heart Foundation, Resuscitation Councils of Southern Africa). Circulation 2004;110:3385-97.

11. Hasegawa K, Hiraide A, Chang $Y$, et al. Association of prehospital advanced airway management with neurologic outcome and survival in patients with out-of-hospital cardiac arrest. JAMA 2013;309:257-66.

12. Hasegawa K, Tsugawa $\mathrm{Y}$, Camargo CA, Jr., et al. Regional variability in survival outcomes of out-of-hospital cardiac arrest: the All-Japan Utstein Registry. Resuscitation 2013;84:1099-107.

13. Messinger-Rapport BJ, Kamel HK. Predictors of do not resuscitate orders in the nursing home. J Am Med Dir Assoc 2005;6:18-21.

14. Ministry of Internal Affairs and Communications. Population data by prefecture. Secondary population data by prefecture. http://www. e-stat.go.jp/SG1/estat/List.do?bid=000001039703

15. Ikegami N, Yoo B-K, Hashimoto $\mathrm{H}$, et al. Japanese universal health coverage: evolution, achievements, and challenges. Lancet 2011;378:1106-15.

16. Ministry of Health Labour and Welfare. Health expenditure database. Secondary health expenditure database. http://www.mhlw.go.jp/ bunya/iryouhoken/iryouhoken03/01.html 
17. Ministry of Internal Affairs and Communications. e-Stat: Portal Site of Official Statistics of Japan. Secondary e-Stat: Portal Site of Official Statistics of Japan. http://www.e-stat.go.jp/SG1/estat/ eStatTopPortalE.do

18. Aylin P, Bottle A, Majeed A. Use of administrative data or clinical databases as predictors of risk of death in hospital: comparison of models. BMJ 2007;334:1044.

19. Williams R. Using the margins command to estimate and interpret adjusted predictions and marginal effects. Stata $J$ 2012;12:308.

20. Zhang Y, Baik SH, Fendrick AM, et al. Comparing local and regional variation in health care spending. $N$ Engl $J$ Med 2012;367:1724-31.

21. Robinson WS. Ecological correlations and the behavior of individuals. Am Soc Rev 1950;15:351-7.
22. Liang $\mathrm{K}-\mathrm{Y}$, Zeger SL. Longitudinal data analysis using generalized linear models. Biometrika 1986;73:13-22.

23. Hanley JA, Negassa A, Edwardes MD, et al. Statistical analysis of correlated data using generalized estimating equations: an orientation. Am J Epidemiol 2003;157:364-75.

24. Panageas KS, Schrag D, Riedel E, et al. The effect of clustering of outcomes on the association of procedure volume and surgical outcomes. Ann Intern Med 2003;139:658-65.

25. Hu FB, Goldberg J, Hedeker D, et al. Comparison of population-averaged and subject-specific approaches for analyzing repeated binary outcomes. Am J Epidemiol 1998;147:694-703.

26. Statistics Bureau MolAaC. Prefectural accounts: economic growth rate and prefectural income per capita. Tokyo, Japan: Japan Statistical Yearbook 2015, 2015. 